\title{
On a new subclass of Ruscheweyh-type harmonic multivalent functions
}

Elif Yaşar* and Sibel Yalçın

Dedicated to Professor Hari M Srivastava

"Correspondence:

elifyasar@uludag.edu.tr

Department of Mathematics,

Faculty of Arts and Science, Uludag

University, Bursa, 16059, Turkey

\begin{abstract}
We introduce a certain subclass of harmonic multivalent functions defined by using a Ruscheweyh derivative operator. We obtain coefficient conditions, distortion bounds, extreme points, convex combination for the above class of harmonic multivalent functions. We also derive inclusion relationships involving the neighborhoods of harmonic multivalent functions belonging to this subclass.
\end{abstract}

MSC: $30 C 45 ; 30 C 50$

Keywords: harmonic; multivalent; Ruscheweyh derivative operator; neighborhood

\section{Introduction}

A continuous function $f=u+i v$ is a complex-valued harmonic function in a domain $D \subset \mathbb{C}$ if both $u$ and $v$ are real harmonic in $D$. In any simply connected domain $D$, we can write $f=h+\bar{g}$, where $h$ and $g$ are analytic in $D$. We call $h$ the analytic part and $g$ the co-analytic part of $f$. The harmonic function $f=h+\bar{g}$ is sense preserving and locally one-to-one in $D$ if $\left|h^{\prime}(z)\right|>\left|g^{\prime}(z)\right|$ in $D$. See Clunie and Sheil-Small [1].

For $p \geq 1, n \in \mathbb{N}$, denote by $\operatorname{SH}(n, p)$ the class of functions $f=h+\bar{g}$ that are sense preserving, harmonic multivalent in the unit disk $U=\{z:|z|<1\}$, where $h$ and $g$ are defined by

$$
h(z)=z^{p}+\sum_{k=n+p}^{\infty} a_{k} z^{k}, \quad g(z)=\sum_{k=n+p-1}^{\infty} b_{k} z^{k}, \quad\left|b_{p}\right|<1,
$$

which are analytic and multivalent functions in $U$.

Also, denote by $\overline{S H}(n, p)$ the subclass of $S H(n, p)$ consisting of harmonic functions $f=$ $h+\bar{g}$, where $h$ and $g$ are of the form

$$
h(z)=z^{p}-\sum_{k=n+p}^{\infty} a_{k} z^{k}, \quad g(z)=-\sum_{k=n+p-1}^{\infty} b_{k} z^{k}, \quad a_{k}, b_{k} \geq 0 .
$$

Note that $\overline{S H}(n, p)$ reduces to $S(n, p)$, the class of analytic multivalent functions with negative coefficients, if the co-analytic part of $f=h+\bar{g}$ is identically zero.

(C) 2013 Yaşar and Yalçın; licensee Springer. This is an Open Access article distributed under the terms of the Creative Commons Attribution License (http://creativecommons.org/licenses/by/2.0), which permits unrestricted use, distribution, and reproduction in any medium, provided the original work is properly cited. 
We define an extended linear derivative operator of a Ruscheweyh-type harmonic function $f=h+\bar{g}$ in $S H(n, p)$ by

$$
D^{\mu, p} f(z)=D^{\mu, p} h(z)+\overline{D^{\mu, p} g(z)}
$$

where $D$ is the Ruscheweyh derivative [2] of power series $\phi(z)=z^{p}+\sum_{k=p+1}^{\infty} \phi_{k} z^{k}$, given by

$$
\begin{aligned}
D^{\mu, p} \phi(z) & =\frac{z^{p}}{(1-z)^{\mu+p}} * \phi(z) \\
& =z^{p}+\sum_{k=p+1}^{\infty}\left(\begin{array}{c}
k+\mu-1 \\
k-p
\end{array}\right) \phi_{k} z^{k} \\
& =z^{p}+\sum_{k=p+1}^{\infty} \frac{\Gamma(k+\mu)}{(k-p) ! \Gamma(p+\mu)} \phi_{k} z^{k} \quad(\mu>-p) .
\end{aligned}
$$

The operator $*$ stands for the Hadamard product or convolution of two power series

$$
\phi(z)=z^{p}+\sum_{k=n+p}^{\infty} \phi_{k} z^{k} \quad \text { and } \quad \Psi(z)=z^{p}+\sum_{k=n+p}^{\infty} \Psi_{k} z^{k}
$$

defined by

$$
(\phi * \Psi)(z)=\phi(z) * \Psi(z)=z^{p}+\sum_{k=n+p}^{\infty} \phi_{k} \Psi_{k} z^{k}
$$

Raina and Srivastava [3] introduced this extended Ruscheweyh operator for the class $S(n, 1)$.

Next, we define the ordinary differential operator $\left(D^{\mu, p} f(z)\right)^{(q)}$ to be

$$
\left(D^{\mu, p} f(z)\right)^{(q)}=\left(D^{\mu, p} h(z)\right)^{(q)}+\overline{\left(D^{\mu, p} g(z)\right)^{(q)}}
$$

where

$$
\begin{aligned}
& \left(D^{\mu, p} h(z)\right)^{(q)}=\frac{p !}{(p-q) !} z^{p-q}+\sum_{k=n+p}^{\infty} \frac{\Gamma(k+\mu)}{(k-p) ! \Gamma(p+\mu)} \frac{k !}{(k-q) !} a_{k} z^{k-q} \text { and } \\
& \left(D^{\mu, p} g(z)\right)^{(q)}=\sum_{k=n+p-1}^{\infty} \frac{\Gamma(k+\mu)}{(k-p) ! \Gamma(p+\mu)} \frac{k !}{(k-q) !} b_{k} z^{k-q}, \quad p>q, p \in \mathbb{N}, q \in \mathbb{N}_{0}=\mathbb{N} \cup\{0\} .
\end{aligned}
$$

Let $S H_{\mu}^{q}(n, p, \lambda, \alpha)$ denote the subclass of $S H(n, p)$ consisting of functions $f=h+\bar{g} \in$ $\operatorname{SH}(n, p)$ that satisfy the condition

$$
\begin{aligned}
& \operatorname{Re}\left\{\frac{\lambda z\left(D^{\mu, p} f(z)\right)^{(q+1)}+(1-\lambda) z\left(D^{1+\mu, p} f(z)\right)^{(q+1)}}{\lambda\left(D^{\mu, p} f(z)\right)^{(q)}+(1-\lambda)\left(D^{1+\mu, p} f(z)\right)^{(q)}}\right\} \geq \alpha(p-q) \\
& \left(0 \leq \lambda \leq 1,0 \leq \alpha<1, p>q, p \in \mathbb{N}, q \in \mathbb{N}_{0}=\mathbb{N} \cup\{0\}, \mu>-p, n \in \mathbb{N}, z \in U\right) .
\end{aligned}
$$

Define $\overline{S H}_{\mu}^{q}(n, p, \lambda, \alpha):=S H_{\mu}^{q}(n, p, \lambda, \alpha) \cap \overline{S H}(n, p)$. 
Taking the co-analytic part of $f=h+\bar{g}$ identically zero and specializing the parameters, we obtain the following subclasses:

(i) $S H_{\mu}^{0}(n, 1, \lambda, 1-\alpha) \supset K_{\mu}(n, \lambda, \alpha)$ (Irmak et al. [4]),

(ii) $S H_{\mu}^{q}(n, p, \lambda,(1-\alpha)(p-q)) \supset T_{\mu}^{q}(n, p, \lambda, \alpha)$ (Ashwah [5]),

(iii) $S H_{\mu}^{0}(n, 1,1,1-\alpha|\gamma|) \supset S_{n}(\gamma, \mu, \alpha)(\gamma \in \mathbb{C} \backslash\{0\})$ (Murugusundaramoorthy and Srivastava [6]).

We will use the notations

$$
\begin{aligned}
& \Omega(i, q):=\frac{i !}{(i-q) !}, \quad i \in\{p, k\}, \\
& C^{p}(k, \mu):=\frac{\Gamma(k+\mu)}{(k-p) ! \Gamma(p+\mu)} .
\end{aligned}
$$

Following Goodman [7] and Ruscheweyh [8] (see also [9-11] and [12]), for $\delta \geq 0$, we define the set of the $\delta$-neighborhood of $f=h+\bar{g} \in \overline{S H}(n, p)$,

$$
\begin{aligned}
N_{n, p}^{\delta}\left(f^{(q)} ; s^{(q)}\right)= & \left\{s \in \overline{S H}(n, p): s(z)=z^{p}-\sum_{k=n+p}^{\infty} A_{k} z^{k}-\sum_{k=n+p-1}^{\infty} B_{k} \bar{z}^{k},\right. \\
& \sum_{k=n+p}^{\infty} k \Omega(k, q)\left(\left|a_{k}-A_{k}\right|+\left|b_{k}-B_{k}\right|\right) \\
& \left.+(n+p-1) \Omega(n+p-1, q)\left|b_{n+p-1}-B_{n+p-1}\right| \leq \delta\right\} .
\end{aligned}
$$

In particular, for the function $e(z)=z^{p}$, we immediately have

$$
\begin{aligned}
N_{n, p}^{\delta}\left(e^{(q)} ; s^{(q)}\right)= & \left\{s \in \overline{S H}(n, p): s(z)=z^{p}-\sum_{k=n+p}^{\infty} A_{k} z^{k}-\sum_{k=n+p-1}^{\infty} B_{k} \bar{z}^{k},\right. \\
& \left.\sum_{k=n+p}^{\infty} k \Omega(k, q)\left(A_{k}+B_{k}\right)+(n+p-1) \Omega(n+p-1, q) B_{n+p-1} \leq \delta\right\} .
\end{aligned}
$$

Ruscheweyh-type harmonic univalent functions have been studied by several authors such as $[13,14]$ and $[15]$. The object of the present paper is to investigate the various properties of multivalent harmonic functions belonging to the subclass $\overline{S H}_{\mu}^{q}(n, p, \lambda, \alpha)$. This class is motivated by two earlier investigations [5] and [3]. We extend the results of [5] which include harmonic multivalent functions. Necessary and sufficient coefficient conditions, distortion bounds, extreme points and convex combination of the above mentioned class are derived. Also, inclusion relationships involving the $(n, \delta)$ neighborhoods of multivalent harmonic functions belonging to this subclass are established.

\section{Main results}

Denote by $S H^{*}(n, p)$ the class of functions $f=h+\bar{g}$ of the form (1) which are sense preserving and multivalent harmonic starlike, satisfying the condition $\frac{\partial}{\partial \theta}\left(\arg f\left(r e^{i \theta}\right)\right) \geq 0$, for each $z=r e^{i \theta}, 0 \leq \theta<2 \pi$, and $0 \leq r<1$. 
Lemma 2.1 Let $f=h+\bar{g}$ be of the form (1). If

$$
\sum_{k=n+p}^{\infty} k\left|a_{k}\right|+\sum_{k=n+p-1}^{\infty} k\left|b_{k}\right| \leq p \quad(p \geq 1, n \in \mathbb{N})
$$

then $f \in S H^{*}(n, p)$.

Remark Lemma 2.1 follows immediately from the result due to Ahuja [16] upon setting $p$ and $k$ instead of $m$ and $n+m-1$, respectively.

Theorem 2.2 Let $f=h+\bar{g}$ be given by (1). Furthermore, let

$$
\begin{aligned}
& \sum_{k=n+p}^{\infty} 2[k+\mu-\lambda(k-p)][k-q-\alpha(p-q)] \frac{C^{p}(k, \mu)}{\Psi \times(p+\mu)} \frac{\Omega(k, q)}{\Omega(p, q)}\left|a_{k}\right| \\
& \quad+\sum_{k=n+p-1}^{\infty}[k+\mu-\lambda(k-p)][(k-q-\alpha(p-q)+1)+|k-q-\alpha(p-q)-1|] \\
& \quad \times\left(\frac{C^{p}(k, \mu)}{\Psi \times(p+\mu)} \frac{\Omega(k, q)}{\Omega(p, q)}\right)\left|b_{k}\right| \leq 1 \\
& \quad\left(0 \leq \lambda \leq 1,0 \leq \alpha<1, p>q, p \in \mathbb{N}, q \in \mathbb{N}_{0}=\mathbb{N} \cup\{0\}, n \in \mathbb{N}, z \in U\right)
\end{aligned}
$$

then $f$ is sense preserving, harmonic multivalent in $U$, and $f \in \operatorname{SH}_{\mu}^{q}(n, p, \lambda, \alpha)$, where

$$
\Psi=[((1-\alpha)(p-q)+1)-|(1-\alpha)(p-q)-1|] .
$$

Proof If the inequality (9) holds for the coefficients of $f=h+\bar{g}$, then by (8), $f$ is sense preserving, harmonic multivalent and starlike in $U$. In view of (5), we need to prove that $\operatorname{Re}\{w\}>0$, where

$$
\begin{aligned}
w= & \left(\lambda z\left(D^{\mu, p} f(z)\right)^{(q+1)}+(1-\lambda) z\left(D^{1+\mu, p} f(z)\right)^{(q+1)}-\alpha(p-q)\left[\lambda\left(D^{\mu, p} f(z)\right)^{(q)}\right.\right. \\
& \left.\left.+(1-\lambda)\left(D^{1+\mu, p} f(z)\right)^{(q)}\right]\right) /\left(\lambda\left(D^{\mu, p} f(z)\right)^{(q)}+(1-\lambda)\left(D^{1+\mu, p} f(z)\right)^{(q)}\right) \\
:= & \frac{A(z)}{B(z)} .
\end{aligned}
$$

Using the fact that $\operatorname{Re} w \geq 0 \Leftrightarrow|1+w| \geq|1-w|$, it suffices to show that

$$
|A(z)+B(z)|-|A(z)-B(z)| \geq 0 .
$$

Therefore we obtain

$$
\begin{aligned}
\mid A(z) & +B(z)|-| A(z)-B(z) \mid \\
\geq & {[\Omega(p, q) \times \Psi]|z|^{p-q} } \\
& \quad-\sum_{k=n+p}^{\infty} 2[k+\mu-\lambda(k-p)][k-q-\alpha(p-q)] \frac{C^{p}(k, \mu) \Omega(k, q)}{(p+\mu)}\left|a_{k}\right||z|^{k-q}
\end{aligned}
$$




$$
\begin{aligned}
& -\sum_{k=n+p-1}^{\infty}([k+\mu-\lambda(k-p)][(k-q-\alpha(p-q)+1)+|k-q-\alpha(p-q)-1|] \\
& \left.\times \frac{C^{p}(k, \mu) \Omega(k, q)}{(p+\mu)}\left|b_{k}\right||z|^{k-q}\right) \\
> & {[\Omega(p, q) \times \Psi]|z|^{p-q} } \\
& \times\left\{1-\sum_{k=n+p}^{\infty} 2[k+\mu-\lambda(k-p)][k-q-\alpha(p-q)] \frac{C^{p}(k, \mu)}{\Psi \times(p+\mu)} \frac{\Omega(k, q)}{\Omega(p, q)}\left|a_{k}\right|\right. \\
& -\sum_{k=n+p-1}^{\infty}([k+\mu-\lambda(k-p)][(k-q-\alpha(p-q)+1)+|k-q-\alpha(p-q)-1|] \\
& \left.\left.\times \frac{C^{p}(k, \mu)}{\Psi \times(p+\mu)} \frac{\Omega(k, q)}{\Omega(p, q)}\left|b_{k}\right|\right)\right\} \\
\geq & 0 .
\end{aligned}
$$

This last expression is non-negative by (9), and so the proof is complete.

Corollary 2.3 For $(1-\alpha)(p-q)<1$ and $n \geq 2$, if the inequality

$$
\begin{aligned}
& \sum_{k=n+p}^{\infty}[k+\mu-\lambda(k-p)][k-q-\alpha(p-q)] \frac{C^{p}(k, \mu)}{(p+\mu)} \frac{\Omega(k, q)}{\Omega(p, q)}\left|a_{k}\right| \\
& \quad+\sum_{k=n+p-1}^{\infty}[k+\mu-\lambda(k-p)][k-q-\alpha(p-q)] \frac{C^{p}(k, \mu)}{(p+\mu)} \frac{\Omega(k, q)}{\Omega(p, q)}\left|b_{k}\right| \\
& \leq(1-\alpha)(p-q) \\
& \quad\left(0 \leq \lambda \leq 1,0 \leq \alpha<1, p>q, p \in \mathbb{N}, q \in \mathbb{N}_{0}=\mathbb{N} \cup\{0\}\right)
\end{aligned}
$$

holds, then $f$ is sense preserving, harmonic multivalent in $U$, and $f \in \operatorname{SH}_{\mu}^{q}(n, p, \lambda, \alpha)$.

Corollary 2.4 For $(1-\alpha)(p-q) \geq 1$, if the inequality

$$
\begin{aligned}
& \sum_{k=n+p}^{\infty}[k+\mu-\lambda(k-p)][k-q-\alpha(p-q)] \frac{C^{p}(k, \mu)}{(p+\mu)} \frac{\Omega(k, q)}{\Omega(p, q)}\left|a_{k}\right| \\
& \quad+\sum_{k=n+p-1}^{\infty}[k+\mu-\lambda(k-p)][k-q-\alpha(p-q)] \frac{C^{p}(k, \mu)}{(p+\mu)} \frac{\Omega(k, q)}{\Omega(p, q)}\left|b_{k}\right| \\
& \leq 1 \\
& \quad\left(0 \leq \lambda \leq 1,0 \leq \alpha<1, p>q, p \in \mathbb{N}, q \in \mathbb{N}_{0}=\mathbb{N} \cup\{0\}\right)
\end{aligned}
$$

holds, then $f$ is sense preserving, harmonic multivalent in $U$, and $f \in \operatorname{SH}_{\mu}^{q}(n, p, \lambda, \alpha)$.

Theorem 2.5 Let $f=h+\bar{g}$ be given by (2). Then 
(i) for $(1-\alpha)(p-q)<1$ and $n \geq 2, f \in \overline{S H}_{\mu}^{q}(n, p, \lambda, \alpha)$ if and only if

$$
\begin{aligned}
& \sum_{k=n+p}^{\infty}[k+\mu-\lambda(k-p)][k-q-\alpha(p-q)] \frac{C^{p}(k, \mu)}{(p+\mu)} \frac{\Omega(k, q)}{\Omega(p, q)} a_{k} \\
& \quad+\sum_{k=n+p-1}^{\infty}[k+\mu-\lambda(k-p)][k-q-\alpha(p-q)] \frac{C^{p}(k, \mu)}{(p+\mu)} \frac{\Omega(k, q)}{\Omega(p, q)} b_{k} \\
& \leq(1-\alpha)(p-q),
\end{aligned}
$$

(ii) for $(1-\alpha)(p-q) \geq 1, f \in \overline{S H}_{\mu}^{q}(n, p, \lambda, \alpha)$ if and only if

$$
\begin{aligned}
& \sum_{k=n+p}^{\infty}[k+\mu-\lambda(k-p)][k-q-\alpha(p-q)] \frac{C^{p}(k, \mu)}{(p+\mu)} \frac{\Omega(k, q)}{\Omega(p, q)} a_{k} \\
& +\sum_{k=n+p-1}^{\infty}[k+\mu-\lambda(k-p)][k-q-\alpha(p-q)] \frac{C^{p}(k, \mu)}{(p+\mu)} \frac{\Omega(k, q)}{\Omega(p, q)} b_{k} \leq 1 .
\end{aligned}
$$

Proof The 'if' part follows from Theorem 2.2, Corollary 2.3 and Corollary 2.4 upon noting that $\overline{S H}_{\mu}^{q}(n, p, \lambda, \alpha) \subset S H_{\mu}^{q}(n, p, \lambda, \alpha)$. For the 'only if' part, we show that $f \notin \overline{S H}_{\mu}^{q}(n, p, \lambda, \alpha)$ if the condition (11) does not hold.

Note that a necessary and sufficient condition for $f=h+\bar{g}$ given by (2) to be in $\overline{S H}_{\mu}^{q}(n, p, \lambda, \alpha)$ is that the condition (5) to be satisfied. This is equivalent to

$$
\begin{aligned}
\operatorname{Re}\{( & (1-\alpha)(p-q)-\sum_{k=n+p}^{\infty}[k+\mu-\lambda(k-p)][k-q-\alpha(p-q)] \\
& \times \frac{C^{p}(k, \mu)}{(p+\mu)} \frac{\Omega(k, q)}{\Omega(p, q)} a_{k} z^{k-p} \\
& \left.-\sum_{k=n+p-1}^{\infty}[k+\mu-\lambda(k-p)][k-q-\alpha(p-q)] \frac{C^{p}(k, \mu)}{(p+\mu)} \frac{\Omega(k, q)}{\Omega(p, q)} b_{k} \bar{z}^{k-p}\right) \\
& /\left(1-\sum_{k=n+p}^{\infty}[k+\mu-\lambda(k-p)] \frac{C^{p}(k, \mu)}{(p+\mu)} \frac{\Omega(k, q)}{\Omega(p, q)} a_{k} z^{k-p}\right. \\
& \left.\left.-\sum_{k=n+p-1}^{\infty}[k+\mu-\lambda(k-p)] \frac{C^{p}(k, \mu)}{(p+\mu)} \frac{\Omega(k, q)}{\Omega(p, q)} b_{k} \bar{z}^{k-p}\right)\right\}
\end{aligned}
$$

$\geq 0$.

The above condition must hold for all values of $z,|z|=r<1$. Upon choosing the values of $z$ on the positive real axis where $0 \leq z=r<1$, we must have

$$
\begin{gathered}
\left((1-\alpha)(p-q)-\sum_{k=n+p}^{\infty}[k+\mu-\lambda(k-p)][k-q-\alpha(p-q)] \frac{C^{p}(k, \mu)}{(p+\mu)} \frac{\Omega(k, q)}{\Omega(p, q)} a_{k} r^{k-p}\right. \\
\left.-\sum_{k=n+p-1}^{\infty}[k+\mu-\lambda(k-p)][k-q-\alpha(p-q)] \frac{C^{p}(k, \mu)}{(p+\mu)} \frac{\Omega(k, q)}{\Omega(p, q)} b_{k} r^{k-p}\right)
\end{gathered}
$$




$$
\begin{aligned}
& \quad /\left(1-\sum_{k=n+p}^{\infty}[k+\mu-\lambda(k-p)] \frac{C^{p}(k, \mu)}{(p+\mu)} \frac{\Omega(k, q)}{\Omega(p, q)} a_{k} r^{k-p}\right. \\
& \left.-\sum_{k=n+p-1}^{\infty}[k+\mu-\lambda(k-p)] \frac{C^{p}(k, \mu)}{(p+\mu)} \frac{\Omega(k, q)}{\Omega(p, q)} b_{k} r^{k-p}\right) \\
& \geq 0 .
\end{aligned}
$$

If the condition (11) does not hold, then the numerator of (12) is negative for $r$ sufficiently close to 1 because of conditions (i) or (ii). Thus there exists a $z_{0}=r_{0}$ in $(0,1)$, for which the quotient in (12) is negative. This contradicts the required condition for $f \in \overline{S H}_{\mu}^{q}(n, p, \lambda, \alpha)$ and so the proof is complete.

Next we determine the distortion bounds for the functions in $\overline{S H}_{\mu}^{q}(n, p, \lambda, \alpha)$.

Theorem 2.6 Let $f \in \overline{S H}_{\mu}^{q}(n, p, \lambda, \alpha)$. Then for $|z|=r<1$, we have

(i) for $(1-\alpha)(p-q)<1$ and $n \geq 2$,

$$
\begin{aligned}
|f(z)| \leq & \left(1+b_{n+p-1}\right) r^{p}+\left(\frac{(1-\alpha)(p-q)}{[n(1-\lambda)+p+\mu][n+(1-\alpha)(p-q)]}\right. \\
& \times \frac{(p+\mu)}{C^{p}(n+p, \mu)} \frac{\Omega(p, q)}{\Omega(n+p, q)} \\
& -\frac{[n(1-\lambda)+p+\mu+\lambda-1][n-1+(1-\alpha)(p-q)]}{[n(1-\lambda)+p+\mu][n+(1-\alpha)(p-q)]} \\
& \left.\times \frac{n(n+p-q)}{(n+p+\mu-1)(n+p)} b_{n+p-1}\right) r^{n+p}
\end{aligned}
$$

and

$$
\begin{aligned}
|f(z)| \geq & \left(1-b_{n+p-1}\right) r^{p}-\left(\frac{(1-\alpha)(p-q)}{[n(1-\lambda)+p+\mu][n+(1-\alpha)(p-q)]}\right. \\
& \times \frac{(p+\mu)}{C^{p}(n+p, \mu)} \frac{\Omega(p, q)}{\Omega(n+p, q)} \\
& -\frac{[n(1-\lambda)+p+\mu+\lambda-1][n-1+(1-\alpha)(p-q)]}{[n(1-\lambda)+p+\mu][n+(1-\alpha)(p-q)]} \\
& \left.\times \frac{n(n+p-q)}{(n+p+\mu-1)(n+p)} b_{n+p-1}\right) r^{n+p},
\end{aligned}
$$

(ii) for $(1-\alpha)(p-q) \geq 1$,

$$
\begin{aligned}
|f(z)| \leq & \left(1+b_{n+p-1}\right) r^{p}+\left(\frac{1}{[n(1-\lambda)+p+\mu][n+(1-\alpha)(p-q)]}\right. \\
& \times \frac{(p+\mu)}{C^{p}(n+p, \mu)} \frac{\Omega(p, q)}{\Omega(n+p, q)} \\
& -\frac{[n(1-\lambda)+p+\mu+\lambda-1][n-1+(1-\alpha)(p-q)]}{[n(1-\lambda)+p+\mu][n+(1-\alpha)(p-q)]} \\
& \left.\times \frac{n(n+p-q)}{(n+p+\mu-1)(n+p)} b_{n+p-1}\right) r^{n+p}
\end{aligned}
$$


and

$$
\begin{aligned}
|f(z)| \geq & \left(1-b_{n+p-1}\right) r^{p}-\left(\frac{1}{[n(1-\lambda)+p+\mu][n+(1-\alpha)(p-q)]}\right. \\
& \times \frac{(p+\mu)}{C^{p}(n+p, \mu)} \frac{\Omega(p, q)}{\Omega(n+p, q)} \\
& -\frac{[n(1-\lambda)+p+\mu+\lambda-1][n-1+(1-\alpha)(p-q)]}{[n(1-\lambda)+p+\mu][n+(1-\alpha)(p-q)]} \\
& \left.\times \frac{n(n+p-q)}{(n+p+\mu-1)(n+p)} b_{n+p-1}\right) r^{n+p} .
\end{aligned}
$$

Proof (i) We only prove the right-hand inequality. The proof for the left-hand inequality is similar and will be omitted. Let $f \in \overline{S H}_{\mu}^{q}(n, p, \lambda, \alpha)$. Taking the absolute value of $f$, we have

$$
\begin{aligned}
|f(z)| \leq & \left(1+b_{n+p-1}\right) r^{p}+\sum_{k=n+p}^{\infty}\left(a_{k}+b_{k}\right) r^{k} \\
\leq & \left(1+b_{n+p-1}\right) r^{p}+\sum_{k=n+p}^{\infty}\left(a_{k}+b_{k}\right) r^{n+p} \\
= & \left(1+b_{n+p-1}\right) r^{p}+\frac{1}{[n(1-\lambda)+p+\mu][n+(1-\alpha)(p-q)]} \frac{(p+\mu)}{C^{p}(n+p, \mu)} \frac{\Omega(p, q)}{\Omega(n+p, q)} \\
& \times \sum_{k=n+p}^{\infty}[n(1-\lambda)+p+\mu][n+(1-\alpha)(p-q)] \\
& \times \frac{C^{p}(n+p, \mu)}{(p+\mu)} \frac{\Omega(n+p, q)}{\Omega(p, q)}\left(a_{k}+b_{k}\right) r^{n+p} \\
\leq & \left(1+b_{n+p-1}\right) r^{p}+\frac{1}{[n(1-\lambda)+p+\mu][n+(1-\alpha)(p-q)]} \frac{(p+\mu)}{C^{p}(n+p, \mu)} \frac{\Omega(p, q)}{\Omega(n+p, q)} \\
& \times \sum_{k=n+p}^{\infty}[k+\mu-\lambda(k-p)][k-q-\alpha(p-q)] \frac{C^{p}(k, \mu)}{(p+\mu)} \frac{\Omega(k, q)}{\Omega(p, q)}\left(a_{k}+b_{k}\right) r^{n+p} .
\end{aligned}
$$

Using Theorem 2.5(i), we obtain

$$
\begin{aligned}
|f(z)| \leq & \left(1+b_{n+p-1}\right) r^{p}+\left(\frac{(1-\alpha)(p-q)}{[n(1-\lambda)+p+\mu][n+(1-\alpha)(p-q)]}\right. \\
& \times \frac{(p+\mu)}{C^{p}(n+p, \mu)} \frac{\Omega(p, q)}{\Omega(n+p, q)} \\
& -\frac{[n(1-\lambda)+p+\mu+\lambda-1][n-1+(1-\alpha)(p-q)]}{[n(1-\lambda)+p+\mu][n+(1-\alpha)(p-q)]} \\
& \left.\times \frac{n(n+p-q)}{(n+p+\mu-1)(n+p)} b_{n+p-1}\right) r^{n+p} .
\end{aligned}
$$

The proof of the other case is similar and so is omitted.

The following covering result follows from the left-hand inequality in Theorem 2.6. 
Corollary 2.7 Let $f$ of the form (2) be so that $f \in \overline{S H}_{\mu}^{q}(n, p, \lambda, \alpha)$. Then

(i) for $(1-\alpha)(p-q)<1$ and $n \geq 2$,

$$
\begin{aligned}
\{w & :|w|<\left[1-\frac{(1-\alpha)(p-q)}{[n(1-\lambda)+p+\mu][n+(1-\alpha)(p-q)]} \frac{(p+\mu)}{C^{p}(n+p, \mu)} \frac{\Omega(p, q)}{\Omega(n+p, q)}\right. \\
& -([n(1-\lambda)+p+\mu][n+(1-\alpha)(p-q)](n+p+\mu-1)(n+p) \\
& -[n(1-\lambda)+p+\mu+\lambda-1][n-1+(1-\alpha)(p-q)]) \\
& \times([n(1-\lambda)+p+\mu][n+(1-\alpha)(p-q)])^{-1} \\
& \left.\left.\times \frac{n(n+p-q)}{(n+p+\mu-1)(n+p)} b_{n+p-1}\right]\right\} \subset f(U),
\end{aligned}
$$

(ii) for $(1-\alpha)(p-q) \geq 1$,

$$
\begin{aligned}
& \left\{w:|w|<\left[1-\frac{1}{[n(1-\lambda)+p+\mu][n+(1-\alpha)(p-q)]} \frac{(p+\mu)}{C^{p}(n+p, \mu)} \frac{\Omega(p, q)}{\Omega(n+p, q)}\right.\right. \\
& -([n(1-\lambda)+p+\mu][n+(1-\alpha)(p-q)](n+p+\mu-1)(n+p) \\
& \quad-[n(1-\lambda)+p+\mu+\lambda-1][n-1+(1-\alpha)(p-q)]) \\
& \quad \times([n(1-\lambda)+p+\mu][n+(1-\alpha)(p-q)])^{-1} \\
& \left.\left.\quad \times \frac{n(n+p-q)}{(n+p+\mu-1)(n+p)} b_{n+p-1}\right]\right\} \subset f(U) .
\end{aligned}
$$

Theorem 2.8 Let $f$ be given by (2). Then $f \in \overline{S H}_{\mu}^{q}(n, p, \lambda, \alpha)$ if and only if

$$
f(z)=\sum_{k=n+p-1}^{\infty}\left(x_{k} h_{k}(z)+y_{k} g_{k}(z)\right)
$$

where $h_{n+p-1}(z)=z^{p}, h_{k}(z)$, for $k=n+p, n+p+1, \ldots$ is of the form

$$
h_{k}(z)= \begin{cases}z^{p}-\frac{(1-\alpha)(p-q)(p+\mu) \Omega(p, q)}{[k+\mu-\lambda(k-p)][k-q-\alpha(p-q)] C^{p}(k, \mu) \Omega(k, q)} z^{k} ; & (1-\alpha)(p-q)<1 \text { and } n \geq 2, \\ z^{p}-\frac{(p+\mu) \Omega(p, q)}{[k+\mu-\lambda(k-p)][k-q-\alpha(p-q)] C^{p}(k, \mu) \Omega(k, q)} z^{k} ; & (1-\alpha)(p-q) \geq 1\end{cases}
$$

and $g_{k}(z)$, for $k=n+p-1, n+p, \ldots$ is of the form

$$
\begin{aligned}
& g_{k}(z)= \begin{cases}z^{p}-\frac{(1-\alpha)(p-q)(p+\mu) \Omega(p, q)}{[k+\mu-\lambda(k-p)][k-q-\alpha(p-q)]]^{p}(k, \mu) \Omega(k, q)} \bar{z}^{k} ; & (1-\alpha)(p-q)<1 \text { and } n \geq 2, \\
z^{p}-\frac{(p+\mu) \Omega(p, q)}{[k+\mu-\lambda(k-p)][k-q-\alpha(p-q)] C^{p}(k, \mu) \Omega(k, q)} \bar{z}^{k} ; & (1-\alpha)(p-q) \geq 1,\end{cases} \\
& x_{n+p-1} \equiv x_{p}=1-\left(\sum_{k=n+p}^{\infty} x_{k}+\sum_{k=n+p-1}^{\infty} y_{k}\right), \quad x_{k} \geq 0, y_{k} \geq 0 .
\end{aligned}
$$

In particular, the extreme points of $\overline{S H}_{\mu}^{q}(n, p, \lambda, \alpha)$ are $\left\{h_{k}\right\}$ and $\left\{g_{k}\right\}$. 
Proof Suppose $(1-\alpha)(p-q)<1, n \geq 2$, and

$$
\begin{aligned}
f(z)= & \sum_{k=n+p-1}^{\infty}\left(x_{k} h_{k}(z)+y_{k} g_{k}(z)\right) \\
= & z^{p}-\sum_{k=n+p}^{\infty} \frac{(1-\alpha)(p-q)(p+\mu) \Omega(p, q)}{[k+\mu-\lambda(k-p)][k-q-\alpha(p-q)] C^{p}(k, \mu) \Omega(k, q)} x_{k} z^{k} \\
& -\sum_{k=n+p-1}^{\infty} \frac{(1-\alpha)(p-q)(p+\mu) \Omega(p, q)}{[k+\mu-\lambda(k-p)][k-q-\alpha(p-q)] C^{p}(k, \mu) \Omega(k, q)} y_{k} \bar{z}^{k} .
\end{aligned}
$$

Then

$$
\begin{aligned}
\sum_{k=n+p}^{\infty} & \frac{[k+\mu-\lambda(k-p)][k-q-\alpha(p-q)] C^{p}(k, \mu) \Omega(k, q)}{(1-\alpha)(p-q)(p+\mu) \Omega(p, q)} \\
& \times \frac{(1-\alpha)(p-q)(p+\mu) \Omega(p, q)}{[k+\mu-\lambda(k-p)][k-q-\alpha(p-q)] C^{p}(k, \mu) \Omega(k, q)} x_{k} \\
& +\sum_{k=n+p-1}^{\infty} \frac{[k+\mu-\lambda(k-p)][k-q-\alpha(p-q)] C^{p}(k, \mu) \Omega(k, q)}{(1-\alpha)(p-q)(p+\mu) \Omega(p, q)} \\
& \times \frac{(1-\alpha)(p-q)(p+\mu) \Omega(p, q)}{[k+\mu-\lambda(k-p)][k-q-\alpha(p-q)] C^{p}(k, \mu) \Omega(k, q)} y_{k} \\
= & \sum_{k=n+p}^{\infty} x_{k}+\sum_{k=n+p-1}^{\infty} y_{k}=1-x_{p} \leq 1
\end{aligned}
$$

and so $f \in \overline{S H}_{\mu}^{q}(n, p, \lambda, \alpha)$.

Conversely, if $f \in \overline{S H}_{\mu}^{q}(n, p, \lambda, \alpha)$, then

$$
a_{k} \leq \frac{(1-\alpha)(p-q)}{[k+\mu-\lambda(k-p)][k-q-\alpha(p-q)]} \frac{(p+\mu)}{C^{p}(k, \mu)} \frac{\Omega(p, q)}{\Omega(k, q)}
$$

and

$$
b_{k} \leq \frac{(1-\alpha)(p-q)}{[k+\mu-\lambda(k-p)][k-q-\alpha(p-q)]} \frac{(p+\mu)}{C^{p}(k, \mu)} \frac{\Omega(p, q)}{\Omega(k, q)}
$$

Set

$$
\begin{aligned}
& x_{k}=\frac{[k+\mu-\lambda(k-p)][k-q-\alpha(p-q)] C^{p}(k, \mu) \Omega(k, q)}{(1-\alpha)(p-q)(p+\mu) \Omega(p, q)} a_{k} \quad(k=n+p, n+p+1, \ldots), \\
& y_{k}=\frac{[k+\mu-\lambda(k-p)][k-q-\alpha(p-q)] C^{p}(k, \mu) \Omega(k, q)}{(1-\alpha)(p-q)(p+\mu) \Omega(p, q)} b_{k} \quad(k=n+p-1, n+p, \ldots)
\end{aligned}
$$

and

$$
x_{p}=1-\left(\sum_{k=n+p}^{\infty} x_{k}+\sum_{k=n+p-1}^{\infty} y_{k}\right)
$$


where $x_{p} \geq 0$. Then, as required, we obtain

$$
f(z)=x_{p} z^{p}+\sum_{k=n+p}^{\infty} x_{k} h_{k}(z)+\sum_{k=n+p-1}^{\infty} y_{k} g_{k}(z) .
$$

The proof for the case $(1-\alpha)(p-q) \geq 1$ is similar and hence is omitted.

Theorem 2.9 The class $\overline{S H}_{\mu}^{q}(n, p, \lambda, \alpha)$ is closed under convex combinations.

Proof Let $f_{i} \in \overline{S H}_{\mu}^{q}(n, p, \lambda, \alpha)$ for $i=1,2, \ldots$, where $f_{i}$ is given by

$$
f_{i}(z)=z^{p}-\sum_{k=n+p}^{\infty} a_{k_{i}} z^{k}-\sum_{k=n+p-1}^{\infty} b_{k_{i}} \bar{z}^{k} .
$$

Then by (10) and (11),

$$
\begin{aligned}
& \sum_{k=n+p}^{\infty}[k+\mu-\lambda(k-p)][k-q-\alpha(p-q)] \frac{C^{p}(k, \mu)}{(p+\mu)} \frac{\Omega(k, q)}{\Omega(p, q)} a_{k_{i}} \\
& \quad+\sum_{k=n+p-1}^{\infty}[k+\mu-\lambda(k-p)][k-q-\alpha(p-q)] \frac{C^{p}(k, \mu)}{(p+\mu)} \frac{\Omega(k, q)}{\Omega(p, q)} b_{k_{i}} \\
& \leq \begin{cases}(1-\alpha)(p-q) & \text { if }(1-\alpha)(p-q)<1 \text { and } n \geq 2, \\
1 & \text { if }(1-\alpha)(p-q) \geq 1 .\end{cases}
\end{aligned}
$$

For $\sum_{i=1}^{\infty} t_{i}=1,0 \leq t_{i} \leq 1$, the convex combination of $f_{i}$ may be written as

$$
\sum_{i=1}^{\infty} t_{i} f(z)=z^{p}-\sum_{k=n+p}^{\infty}\left(\sum_{i=1}^{\infty} t_{i} a_{k_{i}}\right) z^{k}-\sum_{k=n+p-1}^{\infty}\left(\sum_{i=1}^{\infty} t_{i} b_{k_{i}}\right) \bar{z}^{k} .
$$

Then by (13),

$$
\begin{aligned}
& \sum_{k=n+p}^{\infty}[k+\mu-\lambda(k-p)][k-q-\alpha(p-q)] \frac{C^{p}(k, \mu)}{(p+\mu)} \frac{\Omega(k, q)}{\Omega(p, q)}\left(\sum_{i=1}^{\infty} t_{i} a_{k_{i}}\right) \\
& \quad-\sum_{k=n+p-1}^{\infty}[k+\mu-\lambda(k-p)][k-q-\alpha(p-q)] \frac{C^{p}(k, \mu)}{(p+\mu)} \frac{\Omega(k, q)}{\Omega(p, q)}\left(\sum_{i=1}^{\infty} t_{i} b_{k_{i}}\right) \\
& =\sum_{i=1}^{\infty} t_{i}\left\{\sum_{k=n+p}^{\infty}[k+\mu-\lambda(k-p)][k-q-\alpha(p-q)] \frac{C^{p}(k, \mu)}{(p+\mu)} \frac{\Omega(k, q)}{\Omega(p, q)} a_{k_{i}}\right. \\
& \left.\quad-\sum_{k=n+p-1}^{\infty}[k+\mu-\lambda(k-p)][k-q-\alpha(p-q)] \frac{C^{p}(k, \mu)}{(p+\mu)} \frac{\Omega(k, q)}{\Omega(p, q)} b_{k_{i}}\right\} \\
& \leq \begin{cases}(1-\alpha)(p-q) \sum_{i=1}^{\infty} t_{i}=(1-\alpha)(p-q) & \text { if }(1-\alpha)(p-q)<1 \text { and } n \geq 2, \\
1 \sum_{i=1}^{\infty} t_{i}=1 & \text { if }(1-\alpha)(p-q) \geq 1 .\end{cases}
\end{aligned}
$$

This is the condition required by (10) and (11), and so $\sum_{i=1}^{\infty} t_{i} f_{i}(z) \in \overline{S H}_{\mu}^{q}(n, p, \lambda, \alpha)$. 
Theorem 2.10 Let $s(z) \in \overline{S H}_{\mu}^{q}(n, p, \lambda, \alpha)$, then

$$
\overline{S H}_{\mu}^{q}(n, p, \lambda, \alpha) \subset N_{n, p}^{\delta}\left(e^{(q)} ; s^{(q)}\right),
$$

where $e(z)$ and $s(z)$ are given by (7),

(i) for $(1-\alpha)(p-q)<1$ and $n \geq 2$,

$$
\begin{aligned}
\delta= & \frac{(1-\alpha)(p-q)(p+\mu) \Omega(p, q)(n+p)}{[n(1-\lambda)+p+\mu] C^{p}(n+p, \mu)[n+(1-\alpha)(p-q)]} \\
& -[\Theta-(n+p-1) \Omega(n+p-1, q)] B_{n+p-1},
\end{aligned}
$$

(ii) for $(1-\alpha)(p-q) \geq 1$,

$$
\begin{aligned}
\delta= & \frac{(p+\mu) \Omega(p, q)(n+p)}{[n(1-\lambda)+p+\mu] C^{p}(n+p, \mu)[n+(1-\alpha)(p-q)]} \\
& -[\Theta-(n+p-1) \Omega(n+p-1, q)] B_{n+p-1},
\end{aligned}
$$

where

$$
\Theta=\frac{[n(1-\lambda)+p+\mu+\lambda-1][n-1+(1-\alpha)(p-q)] \Omega(n+p-1, q) n(n+p)}{[n(1-\lambda)+p+\mu](n+p+\mu-1)[n+(1-\alpha)(p-q)]} .
$$

Proof Let $s(z) \in \overline{S H}_{\mu}^{q}(n, p, \lambda, \alpha),(1-\alpha)(p-q)<1$ and $n \geq 2$. We need to show that $s(z) \in$ $N_{n, p}^{\delta}\left(e^{(q)} ; s^{(q)}\right)$. It suffices to show that $s$ satisfies the condition (7). In view of Theorem 2.5(i), we have

$$
\begin{aligned}
& \left(\frac{[n(1-\lambda)+p+\mu] C^{p}(n+p, \mu)}{(p+\mu) \Omega(p, q)}\right) \sum_{k=n+p}^{\infty}(k-q-\alpha(p-q)) \Omega(k, q)\left(A_{k}+B_{k}\right) \\
& \leq(1-\alpha)(p-q) \\
& \quad-\frac{[n(1-\lambda)+p+\mu+\lambda-1][n-1+(1-\alpha)(p-q)] C^{p}(n+p-1, \mu) \Omega(n+p-1, q)}{(p+\mu) \Omega(p, q)} \\
& \quad \times B_{n+p-1} .
\end{aligned}
$$

Then

$$
\begin{aligned}
\sum_{k=n+p}^{\infty} & k \Omega(k, q)\left(A_{k}+B_{k}\right) \\
\leq & \frac{(1-\alpha)(p-q)(p+\mu) \Omega(p, q)}{[n(1-\lambda)+p+\mu] C^{p}(n+p, \mu)} \\
& -\left(\frac{[n(1-\lambda)+p+\mu+\lambda-1][n-1+(1-\alpha)(p-q)] \Omega(n+p-1, q) n}{[n(1-\lambda)+p+\mu](n+p+\mu-1)}\right) B_{n+p-1} \\
& +\frac{(q+\alpha(p-q))}{(n+p)} \sum_{k=n+p}^{\infty} k \Omega(k, q)\left(A_{k}+B_{k}\right)
\end{aligned}
$$


so that

$$
\begin{aligned}
\sum_{k=n+p}^{\infty} k \Omega(k, q)\left(A_{k}+B_{k}\right) & \leq \frac{(1-\alpha)(p-q)(p+\mu) \Omega(p, q)(n+p)}{[n(1-\lambda)+p+\mu] C^{p}(n+p, \mu)[n+(1-\alpha)(p-q)]}-\Theta B_{n+p-1} \\
& =\delta-(n+p-1) \Omega(n+p-1, q) B_{n+p-1},
\end{aligned}
$$

which, in view of definition (7), completes the proof of Theorem 2.10. The proof of other case is similar and so is omitted.

Remark Taking the co-analytic part of $s(z)$ of the form (7) identically zero and letting $s \in S H_{\mu}^{q}(n, p, \lambda,(1-\alpha)(p-q))$, we obtain the neighborhood result of Ashwah [5].

Remark Taking the co-analytic part of $s(z)$ of the form (7) identically zero and letting $s \in S H_{\mu}^{0}(n, 1,1,1-\alpha|\gamma|)$, we obtain the neighborhood result of Murugusundaramoorthy and Srivastava [6].

Theorem 2.11 Let $f \in \overline{S H}_{n, p}^{m}(q, \lambda, \alpha)$ and

(i) for $(1-\alpha)(p-q)<1$ and $n \geq 2$,

$$
\begin{aligned}
\delta \leq & \Omega(n+p-1, q) \\
& \times\left[p-\frac{(1-\alpha)(p-q)(p+\mu) \Omega(p, q)(n+p-q) !}{[n(1-\lambda)+p+\mu][n+(1-\alpha)(p-q)] C^{p}(n+p, \mu)(n+p-1) !}\right. \\
& \left.-((n+p-1)-\Lambda) b_{n+p-1}\right],
\end{aligned}
$$

(ii) for $(1-\alpha)(p-q) \geq 1$,

$$
\begin{aligned}
\delta \leq & \Omega(n+p-1, q) \\
& \times\left[p-\frac{(p+\mu) \Omega(p, q)(n+p-q) !}{[n(1-\lambda)+p+\mu][n+(1-\alpha)(p-q)] C^{p}(n+p, \mu)(n+p-1) !}\right. \\
& \left.-((n+p-1)-\Lambda) b_{n+p-1}\right]
\end{aligned}
$$

then

$$
N_{n, p}^{\delta}\left(f^{(q)} ; s^{(q)}\right) \subset S H^{*}(n, p)
$$

where

$$
\Lambda=\frac{[n(1-\lambda)+p+\mu+\lambda-1][n-1+(1-\alpha)(p-q)] \Omega(n+p-1, q) n(n+p-q) !}{[n(1-\lambda)+p+\mu][n+(1-\alpha)(p-q)](n+p-1) !(n+p+\mu-1)}
$$

Proof Let $(1-\alpha)(p-q)<1$ and $n \geq 2$. Also, suppose that $f(z) \in \overline{S H}_{n, p}^{m}(q, \lambda, \alpha)$ and $s(z) \in$ $N_{n, p}^{\delta}\left(f^{(q)} ; s^{(q)}\right)$. We need to show that $s(z) \in S H^{*}(n, p)$. It suffices to show that $s$ satisfies the 
condition (8). We have

$$
\begin{aligned}
\sum_{k=n+p}^{\infty} & k\left(A_{k}+B_{k}\right)+(n+p-1) B_{n+p-1} \\
\leq & \sum_{k=n+p}^{\infty} k\left[\left|a_{k}-A_{k}\right|+\left|b_{k}-B_{k}\right|\right]+(n+p-1)\left|b_{n+p-1}-B_{n+p-1}\right| \\
& +\sum_{k=n+p}^{\infty} k\left(a_{k}+b_{k}\right)+(n+p-1) b_{n+p-1} \\
\leq & \frac{1}{\Omega(n+p-1, q)}\left[\sum_{k=n+p}^{\infty} k \Omega(k, q)\left(\left|a_{k}-A_{k}\right|+\left|b_{k}-B_{k}\right|\right)\right. \\
& \left.+(n+p-1) \Omega(n+p-1, q)\left|b_{n+p-1}-B_{n+p-1}\right|\right]+(n+p-1) b_{n+p-1} \\
& +\left(\frac{(p+\mu) \Omega(p, q)(n+p-q) !}{[n(1-\lambda)+p+\mu][n+(1-\alpha)(p-q)] C^{p}(n+p, \mu)(n+p-1) !}\right) \\
& \times\left(\sum_{k=n+p}^{\infty}[k+\mu-\lambda(k-p)][k-q-\alpha(p-q)] \frac{C^{p}(k, \mu)}{(p+\mu)} \frac{\Omega(k, q)}{\Omega(p, q)}\left(a_{k}+b_{k}\right)\right) \\
\leq & \frac{\delta}{\Omega(n+p-1, q)}+(n+p-1) b_{n+p-1} \\
& +\left(\frac{(p+\mu) \Omega(p, q)(n+p-q) !}{[n(1-\lambda)+p+\mu][n+(1-\alpha)(p-q)] C^{p}(n+p, \mu)(n+p-1) !}\right) \\
& \times((1-\alpha)(p-q) \\
& -\frac{[n(1-\lambda)+p+\mu+\lambda-1][n-1+(1-\alpha)(p-q)] C^{p}(n+p-1, \mu) \Omega(n+p-1, q)}{(p+\mu) \Omega(p, q)} \\
& \\
& \\
&
\end{aligned}
$$

Now this expression is never greater than $p$ provided that

$$
\begin{aligned}
\delta \leq & \Omega(n+p-1, q)\left[p-\frac{(1-\alpha)(p-q)(p+\mu) \Omega(p, q)(n+p-q) !}{[n(1-\lambda)+p+\mu][n+(1-\alpha)(p-q)] C^{p}(n+p, \mu)(n+p-1) !}\right. \\
& \left.-((n+p-1)-\Lambda) b_{n+p-1}\right] .
\end{aligned}
$$

The proof of the other case is similar and so is omitted.

\section{Competing interests}

The authors declare that they have no competing interests.

\section{Authors' contributions}

Both authors contributed equally and significantly in writing this paper. And they also read and approved the final manuscript.

Received: 5 December 2012 Accepted: 12 May 2013 Published: 30 May 2013 


\section{References}

1. Clunie, J, Sheil-Small, T: Harmonic univalent functions. Ann. Acad. Sci. Fenn., Ser. A 1 Math. 9, 3-25 (1984)

2. Ruscheweyh, S: New criteria for univalent functions. Proc. Am. Math. Soc. 49, 109-115 (1975)

3. Raina, RK, Srivastava, HM: Inclusion and neighborhood properties of some analytic and multivalent functions. J. Inequal. Pure Appl. Math. 7(1), Article 5 (2006)

4. Irmak, H, Joshi, SB, Raina, RK: On certain novel subclasses of analytic and univalent functions. Kyungpook Math. J. 46 , 543-552 (2006)

5. El-Ashwah, RM: Inclusion and neighborhood properties of a certain subclasses of $p$-valent functions with negative coefficients. Filomat 23(3), 1-13 (2009)

6. Murugusundaramoorthy, G, Srivastava, HM: Neighborhoods of certain classes of analytic functions of complex order. J. Inequal. Pure Appl. Math. 5(2), Article 24 (2004)

7. Goodman, AW: Univalent functions and non-analytic curves. Proc. Am. Math. Soc. 8, 598-601 (1957)

8. Ruscheweyh, S: Neighborhoods of univalent functions. Proc. Am. Math. Soc. 81(4), 521-527 (1981)

9. Altıntaş, O, Irmak, H, Srivastava, HM: Neighborhoods for certain subclasses of multivalently analytic functions defined by using a differential operator. Comput. Math. Appl. 55, 331-338 (2008)

10. Bednarz, U, Kanas, S: Stability of the integral convolution of $k$-uniformly convex and k-starlike functions. J. Appl. Anal. 10(1), 105-115 (2004)

11. Frasin, BA: Neighborhoods of certain multivalent functions with negative coefficients. Appl. Math. Comput. 193, 1-6 (2007)

12. Kanas, S, Bednarz, U: Generalized neighbourhoods and stability of convolution for the class of k-uniformly convex and k-starlike functions. Zeszyty Nauk. Politech. Rzeszowskiej Mat. 23, 29-38 (1999)

13. Darus, M, Al-Shaqsi, K: On harmonic univalent functions defined by a generalized Ruscheweyh derivatives operator. Lobachevskii J. Math. 22, 19-26 (2006)

14. Ebadian, A, Tehranchi, A: On certain classes of harmonic $p$-valent functions by applying the Ruscheweyh derivatives. Filomat 23(1), 91-101 (2009)

15. Murugusundaramoorthy, G: A class of Ruscheweyh-type harmonic univalent functions with varying arguments. Southwest J. Pure Appl. Math. 2, 90-95 (2003)

16. Ahuja, OP, Jahangiri, JM: Multivalent harmonic starlike functions. Ann. Univ. Mariae Curie-Skīodowska, Sect. A 55(1), $1-13(2001)$

doi:10.1186/1029-242X-2013-271

Cite this article as: Yaşar and Yalçın: On a new subclass of Ruscheweyh-type harmonic multivalent functions. Journal of Inequalities and Applications 2013 2013:271.

\section{Submit your manuscript to a SpringerOpen ${ }^{\ominus}$ journal and benefit from:}

- Convenient online submission

- Rigorous peer review

- Immediate publication on acceptance

- Open access: articles freely available online

- High visibility within the field

- Retaining the copyright to your article 